Check for updates

Cite this: RSC Adv., 2019, 9, 14766

\title{
Interface depended electronic and magnetic properties of vertical $\mathrm{Crl}_{3} / \mathrm{WSe}_{2}$ heterostructures
}

\author{
Mei Ge, ${ }^{\text {ab }}$ Yan Su, (D) ${ }^{c}$ Han Wang, ${ }^{d}$ Guohui Yang ${ }^{b}$ and Junfeng Zhang (D)*b
}

Received 11th March 2019

Accepted 7th May 2019

DOI: 10.1039/c9ra01825e

rsc.li/rsc-advances

\begin{abstract}
Owing to the great potential applications in information processing and storage, two-dimensional (2D) magnetic materials have recently attracted significant attention. Here, using first-principles calculations, we investigate the electronic and magnetic properties of the van der Waals $\mathrm{Crl}_{3} / \mathrm{WSe}_{2}$ heterostructures. We find that after forming heterostructures, monolayer $\mathrm{Crl}_{3}$ undergoes a direct to indirect band gap transition and its gap size is greatly reduced. In particular, the out-plane spin quantization axis of monolayer $\mathrm{Crl}_{3}$ is tuned into in-plane for most stacking configurations of $\mathrm{Crl}_{3} /$ $\mathrm{WSe}_{2}$. We further reveal that the transition of the easy magnetization direction is mainly originated from the hybridization between $\mathrm{Cr}-\mathrm{d}$ and $\mathrm{Se}-\mathrm{p}$ orbitals. These theoretical results provide a useful picture for the electronic structure and magnetic anisotropy behaviors in vertical $\mathrm{Crl}_{3} / \mathrm{WSe}_{2}$ heterostructures.
\end{abstract}

\section{Introduction}

As triggered by graphene, ${ }^{1,2}$ two-dimensional (2D) materials such as hexagonal $\mathrm{BN}^{3}$ and transition metal dichalcogenides (TMDs), ${ }^{4,5}$ have become the focus of intensive studies during the past decade because of their unique properties and the great promise for novel applications. Among them, materials with long-range ferromagnetic order are most desirable for applications in spintronics devices. ${ }^{6}$ However, most 2D materials in their pristine forms are intrinsically nonmagnetic, and the magnetic moments introduced by dopants, defects, edges, or coupling to ferromagnetic substrates ${ }^{7}$ are mostly very local. Therefore, the recently fabricated $2 \mathrm{D}$ intrinsic ferromagnetic materials, such as chromium germanium telluride $\left(\mathrm{Cr}_{2} \mathrm{Ge}_{2} \mathrm{Te}_{6}\right.$ (ref. 8)), $\mathrm{CrI}_{3}$ (ref. 9) and $\mathrm{VSe}_{2},{ }^{10}$ have attracted much attention for the promised applications as high-density magnetic memories and spintronic applications at the nanoscale.

$\mathrm{CrI}_{3}$ is layered van der Waals material with order ferromagnetically, the Curie temperature $\left(T_{\mathrm{c}}\right)$ is $61 \mathrm{~K}$ for bulk $\mathrm{k}^{11-14}$ and $45 \mathrm{~K}$ for monolayer. ${ }^{9}$ Monolayer $\mathrm{CrI}_{3}$ is a semiconductor ${ }^{9,15}$ with the band gap of $1.2 \mathrm{eV}^{16-18}$ By including spin-orbit coupling (SOC), the band gap will decrease to $0.9 \mathrm{eV} .^{16,18}$ The magnetism arises from the partially filled d orbitals of the $\mathrm{Cr}^{3+}$ ion, and the magnetocrystalline anisotropy favours an out-plane spin

${ }^{a}$ School of Physics and Information Engineering, Shanxi Normal University, Linfen, 041004, China

${ }^{b}$ Key Laboratory of Spectral Measurement and Analysis of Shanxi Province, Shanxi Normal University, Linfen, 041004, China.E-mail: zhangjf@sxnu.edu.cn

${ }^{c}$ Key Laboratory of Materials Modification by Laser, Ion and Electron Beams, Dalian University of Technology, Ministry of Education, Dalian 116024, China

${ }^{d}$ Chemical Sciences Division, Lawrence Berkeley National Laboratory, Berkeley, California 94720, USA orientation. ${ }^{12}$ It has been demonstrated that the magnetic properties of $\mathrm{CrI}_{3}$ can be controlled by strain, ${ }^{19-23}$ electric field $\mathrm{s}^{24-28}$ and magnetic fields. ${ }^{21,29}$

To combine the advantages of two different 2D materials, stacking them into a heterostructure has been proven to be an effective way. ${ }^{30,31}$ Until now, many kinds of $2 \mathrm{D}$ heterostructures have been fabricated successfully in experiments, ${ }^{32-34}$ and their physical properties have been predicated theoretically. ${ }^{35-39}$ For magnetic materials, it is known that they can be integrated with many other materials, including conductors ${ }^{40}$ and semiconductors. ${ }^{41}$ Recently, increasing attention has been paid to the 2D ferromagnetic heterostructures. For example, by the electrostatic doping in $\mathrm{CrI}_{3} /$ graphene heterostructures, Jiang et al. found that the saturation magnetization can be tuned up to $40 \%$ experimentally. ${ }^{25} \mathrm{~A}$ theoretical study ${ }^{16}$ on $\mathrm{CrI}_{3} /$ graphene heterostructure indicated that a Chern insulating state can be achieved. Moreover, it is possible to control the spin and valley pseudospin properties of $\mathrm{WSe}_{2}$ by constructing $\mathrm{CrI}_{3} / \mathrm{WSe}_{2}$ ferromagnetic heterostructure. ${ }^{42,43}$ However, some questions still need to be answered. For example, what are the interfacial (stacking type) effects on the electronic and magnetic properties of this kinds of heterostructures? Is it possible to tune the magnetic anisotropy energy (MAE) in $\mathrm{CrI}_{3} / \mathrm{WSe}_{2}$ heterostructures?

In this paper, by using first principle calculations, we report the interface effects on the electronic structure and magnetic properties in 2D ferromagnetic $\mathrm{CrI}_{3} / \mathrm{WSe}_{2}$ heterostructures. We consider three different stacking types for both bilayer and triple layer vertical heterostructures. The tuneable band gap and magnetic properties can be found and understood with the help of orbital hybridization and SOC effects. 


\section{Computational methods}

The structural and electronic properties of monolayer $\mathrm{CrI}_{3}$, bilayer and triple layer $\mathrm{CrI}_{3} / \mathrm{WSe}_{2}$ heterostructures were computed using the DFT and projector-augmented wave method (PAW) ${ }^{44}$ implemented in the VASP code..$^{45}$ The PerdewBurke-Ernzerhof (PBE) functiona ${ }^{46}$ was used to describe the exchange-correction interaction. The plane wave basis was expanded up to a cutoff energy $550 \mathrm{eV}$. Grimme's semiempirical DFT-D3 scheme for dispersion correction ${ }^{47}$ was employed to describe the van der Waals (vdW) interactions between the $\mathrm{CrI}_{3}$ and the $\mathrm{WSe}_{2}$ layers. In the direction perpendicular to the sheet, $5 \mathrm{~nm}$ thickness was selected to avoid any spurious interaction with the image sheets. For each configuration, the atomic coordinates and in-plane supercell parameters were fully relaxed, until the Hellmann-Feynman force on each atom became smaller than $0.01 \mathrm{eV} \AA^{-1}$ and the total energy was converged to be within $10^{-6} \mathrm{eV}$. The $2 \mathrm{D}$ Brillouin zones were sampled by an $14 \times 14 \times 1 k$-point grid mesh. ${ }^{48} \mathrm{Spin}$ polarization was taken into the account, and the effect of SOC was also introduced for the calculations of the electric properties. In addition, to include the interactions between $3 \mathrm{~d}$ orbits of $\mathrm{Cr}$ atoms, the DFT+U methods described by Dudarev ${ }^{49}$ were used. Here, in the electronic calculations, the on-site Coulomb parameter $(U)$ and the exchange parameter $(J)$ were selected as $U$ $=2.7 \mathrm{eV}$ and $J=0.7 \mathrm{eV},{ }^{17,18}$ respectively.

\section{Results and discussion}

As shown in Fig. 1a, monolayer $\mathrm{CrI}_{3}$ is a $\mathrm{I}-\mathrm{Cr}-\mathrm{I}$ sandwich structure where each $\mathrm{Cr}$ is surrounded by six first neighboring I atoms. In the $\mathrm{Cr}$ atomic layer, the $\mathrm{Cr}$ atoms form a honeycomb lattice. Owing to the octahedral symmetry, the five d-orbitals $\left(\mathrm{d}_{x y}, \mathrm{~d}_{x z}, \mathrm{~d}_{y z}, \mathrm{~d}_{x^{2}-y^{2}}, \mathrm{~d}_{z}{ }^{2}\right)$ of $\mathrm{Cr}$ are split into three half-filled $\mathrm{t}_{2 \mathrm{~g}}$ orbitals and two empty $\mathrm{e}_{g}$ orbitals. ${ }^{17}$ The magnetization comes directly from the honeycomb $\mathrm{Cr}$ lattice. The orbital configuration of the $\mathrm{I}^{-}$ion $\left(5 \mathrm{~s}^{2} 5 \mathrm{p}^{6}\right)$ has a very strong SOC, which results in antisymmetric exchange effects and anisotropic symmetric
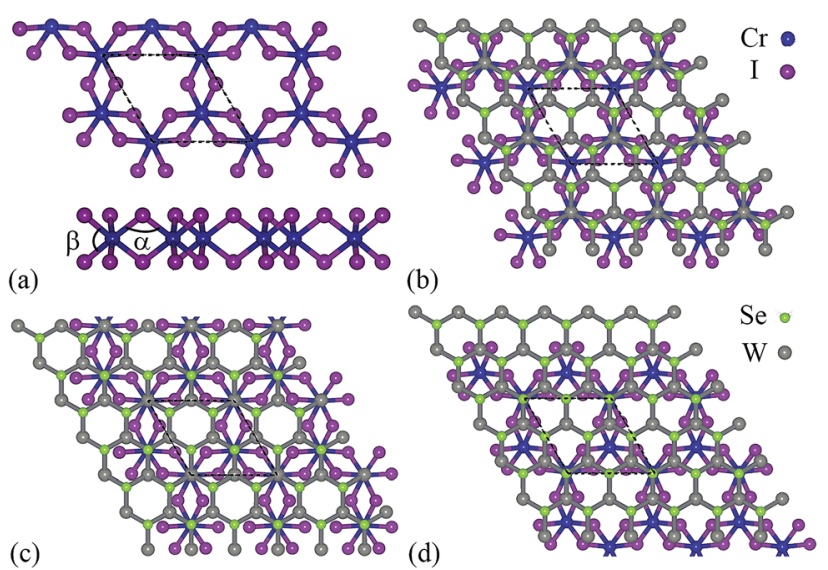

Fig. 1 The structure model for (a) monolayer $\mathrm{Crl}_{3}$ (upper: top view lower: side view), and (b-d) $\mathrm{Crl}_{3} / \mathrm{WSe}_{2}$ heterostructures with different stacking way ((b) Se-site, (c) top-site and (d) W-site). exchange effects. ${ }^{17}$ In Fig. 2, we show the band structures of monolayer $\mathrm{CrI}_{3}$ without and with SOC, where the direct band gaps are $1.05 \mathrm{eV}$ and $0.72 \mathrm{eV}$, respectively. These results are consistent with previous DFT calculations. ${ }^{16-18}$

We discuss here the bilayer $\mathrm{CrI}_{3} / \mathrm{WSe}_{2}$ and trilayer $\mathrm{WSe}_{2} / \mathrm{CrI}_{3} /$ $\mathrm{WSe}_{2}$ heterostructures. For monolayer $\mathrm{CrI}_{3}$ and $\mathrm{WSe}_{2}$, the optimized lattice parameter is $7.002 \AA$ and $3.321 \AA$, respectively. In bilayer $\mathrm{CrI}_{3} / \mathrm{WSe}_{2}$ heterostructure, monolayer $\mathrm{CrI}_{3}(1 \times 1)$ stacked on monolayer $\mathrm{WSe}_{2}(2 \times 2)$ vertically. The trilayer $\mathrm{WSe}_{2} /$ $\mathrm{CrI}_{3} / \mathrm{WSe}_{2}$ heterostructure has a sandwich structure, in which the monolayer $\mathrm{CrI}_{3}(1 \times 1)$ is surrounded by two layers of $\mathrm{WSe}_{2}$ $(2 \times 2)$. Therefore, the lattice mismatch between $\mathrm{CrI}_{3}(1 \times 1)$ and $\mathrm{WSe}_{2}(2 \times 2)$ is about $5.42 \%$. In both bilayer and triple layer heterostructures, the lattice parameter of adopted that of $\mathrm{CrI}_{3}$ lattice $(7.002 \AA)$, and the $\mathrm{WSe}_{2}$ layer has been enlarged uniformly. As shown in Fig. 1b-d, three kinds of vertical stacking types have been considered in the present calculation, bi-Se, bi-T and bi-W for bilayer heterostructures. For bi-Se $\mathrm{CrI}_{3} /$ $\mathrm{WSe}_{2}$ heterostructure, as shown in Fig. $1 \mathrm{~b}$, only one $\mathrm{Cr}$ atom is located on the top of a W atom in a unit cell. Similarly, for the bi$\mathrm{W}$ configuration, one $\mathrm{Cr}$ atom is located on the top of a $\mathrm{I}_{2}$ pair in a unit cell (see Fig. 1d). For the bi-T configuration as shown in Fig. 1c, one $\mathrm{Cr}$ atom is located on the top of $\mathrm{W}$ atom, and the other $\mathrm{Cr}$ atom located on the top of $\mathrm{I}_{2}$ atom. In the trilayer $\mathrm{WSe}_{2} /$ $\mathrm{CrI}_{3} / \mathrm{WSe}_{2}$ heterostructures, the $\mathrm{CrI}_{3} / \mathrm{WSe}_{2}$ stacking in tri-Se, tri$\mathrm{T}$ and tri-W configurations are similar to those in bi-Se, bi-T and bi-W configurations, and the two $\mathrm{WSe}_{2}$ layers are stacked by the AA type.

As can be seen from the data in Table 1, the bi-Se (tri-Se) stacking type is the most favourite configuration in the bilayer (trilayer) heterostructures, which is 6.33 (23.19) and 85.40 (18.76) meV per cell more stable than bi-T (tri-T) and bi-W (triW). Meanwhile, the -Se configuration also has the shortest interlayer distance ( $0.6654 \mathrm{~nm}$ for bi-Se, $0.6667 \mathrm{~nm}$ for tri-Se), compared with those of $-\mathrm{T}(0.6674 \mathrm{~nm}$ for bi-T and $0.6668 \mathrm{~nm}$ for tri-T) and $-\mathrm{W}(0.6685 \mathrm{~nm}$ for bi-W and 0.6842 for tri-W) configurations. Even so, it is noted that the energy differences between different stacking configurations are relatively small and their interlayer distances are comparable, suggesting the superlubricity in the vertical $\mathrm{CrI}_{3} / \mathrm{WSe}_{2}$ heterostructures, which has been found in bilayer graphene. ${ }^{50}$ Moreover, the Bader charger analysis ${ }^{51}$ suggests that, the -Se configurations have less

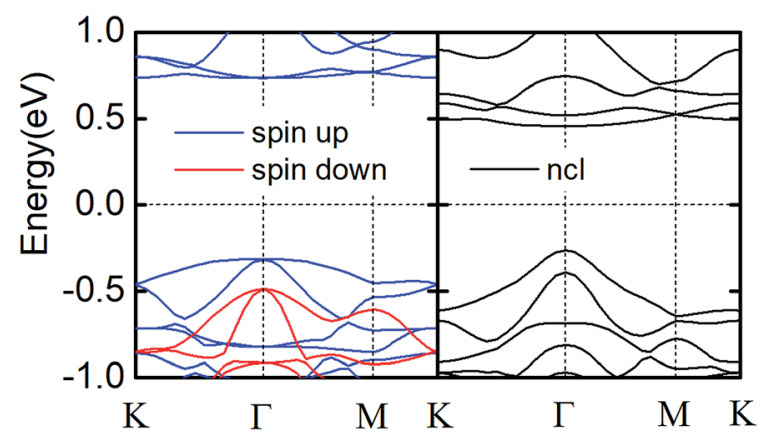

Fig. 2 Band structure of monolayer $\mathrm{Crl}_{3}$ without (left panel) and with (right panel) SOC. 
Table 1 The calculated relative energy $\left(E_{r}\right.$, meV per cell), inter-layer distance $(d, \mathrm{~nm})$ and the inter-layer charge transfer $(Q$, electrons per cell) for bi-layer and tri-layer $\mathrm{Crl}_{3} / \mathrm{WSe}_{2}$ heterostructures. SOC has been included in all calculations

\begin{tabular}{llll}
\hline & $E_{\mathrm{r}}$ & $d$ & $Q$ \\
\hline Bi-Se & 0 & 0.6654 & 0.009 \\
Bi-T & 6.33 & 0.6674 & 0.012 \\
Bi-W & 85.40 & 0.6685 & 0.014 \\
Tri-Se & 0 & 0.6667 & 0.007 \\
Tri-T & 23.19 & 0.6668 & 0.015 \\
Tri-W & 18.76 & 0.6842 & 0.023 \\
\hline
\end{tabular}

inter-layer charge transfer (0.009 electrons per cell for bi-Se, and 0.007 electrons per cell for tri-Se) than -T and -W configurations. The electronic properties and magnetic properties of $\mathrm{CrI}_{3}$ are known to rely on its structural properties. Fig. 3 shows that, the distribution of the bond length (Cr-I, $\left.L_{\mathrm{bond}}\right)$ and bond angels $(\alpha$ : $\angle \mathrm{I}-\mathrm{Cr}-\mathrm{I}, \beta: \angle \mathrm{Cr}-\mathrm{I}-\mathrm{Cr}$ as labelled in Fig. 1 a) of $\mathrm{CrI}_{3}$ in monolayer (black dots), bilayer (left panel) and trilayer (right panel) heterostructures. For the case of monolayer $\mathrm{CrI}_{3}$, according to our calculation, the lattice constant, $L_{\text {bond }}, \alpha$ and $\beta$ are $7.002 \AA$, $2.737 \AA, 90.6^{\circ}$ and $94.5^{\circ}\left(84.8^{\circ}\right)$, respectively. These results are consistent with previous theoretical results. ${ }^{12,13,19,24}$ When forming $\mathrm{CrI}_{3} / \mathrm{WSe}_{2}$ heterostructures, the inter-layer van de Waals interaction makes both the bond length and the bond angles of $\mathrm{CrI}_{3}$ slightly changed. As shown in Fig. 3, the bond information of $\mathrm{CrI}_{3}$ in bi-Se and tri-Se are closer with the monolayer case, which are responsible for the lower formation

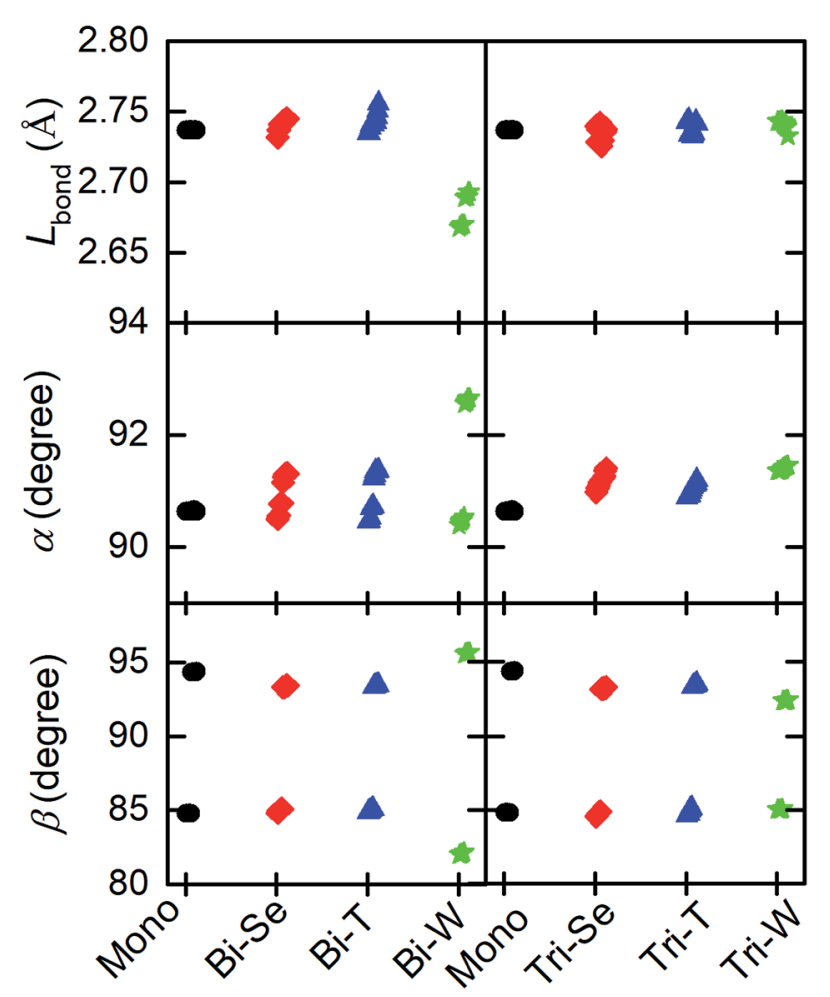

Fig. 3 Bond length ( $\left.L_{\text {bond }}\right)$ and bond angle $(\alpha$ and $\beta$ ) for bilayer and trilayer $\mathrm{Crl}_{3} / \mathrm{WSe}_{2}$ heterostructures with three different stacking models. energy indicated in Table 1. Nevertheless, both the bond angle and bond length have been interrupted by the $\mathrm{WSe}_{2}$ layer in bilayer and triple layer $\mathrm{CrI}_{3} / \mathrm{WSe}_{2}$ heterostructures. The bond angle of $\alpha$ and $\beta$ accounts the ferromagnetic superexchange symmetry. ${ }^{24}$ Therefore, the electronic properties and magnetic properties of $\mathrm{CrI}_{3}$ in heterostructure are supposed to be different from those of monolayer $\mathrm{CrI}_{3}$.

We then next discuss the electronic and magnetic properties of the $\mathrm{CrI}_{3} / \mathrm{WSe}_{2}$ heterostructures. When stacked into heterostructure, as shown in Fig. 4 for bilayer and Fig. 5 for triple layer $\mathrm{CrI}_{3} / \mathrm{WSe}_{2}$ heterostructures, the band structure of $\mathrm{CrI}_{3}$ depends on the interlayer interactions. Firstly, we can also see from the band structures is that, the band alignment in both bilayer and triple layer $\mathrm{CrI}_{3} / \mathrm{WSe}_{2}$ heterostructures can be categorized into staggered type (type-II). Secondly, compared with the band gap of monolayer $\mathrm{CrI}_{3}$ (see Fig. 2), i.e., $1.05 \mathrm{eV}$ (without SOC) and $0.72 \mathrm{eV}$ (with SOC), band gap of $\mathrm{CrI}_{3}$ in heterostructures is larger. Without (with) SOC, band gap of $\mathrm{CrI}_{3}$ in bilayer heterostructures is 1.13 (1.12), 1.13 (1.07) and $1.19(0.90) \mathrm{eV}$ for bi-Se, bi-T and bi-W, respectively. For trilayer $\mathrm{CrI}_{3} / \mathrm{WSe}_{2}$ heterostructures, the band gaps without (with) SOC are 1.07 (1.07) eV for tri-Se, 1.07 (1.04) eV for tri-T and 0.96 (1.01) eV for tri-W, respectively (see Fig. 5). Thirdly, the direct-to-indirect band gap transition can be found from monolayer $\mathrm{CrI}_{3}$ to $\mathrm{CrI}_{3} / \mathrm{WSe}_{2}$ heterostructures. For monolayer $\mathrm{CrI}_{3}$, either with or without SOC (see Fig. 2), the band gap is direct. However, for both bilayer and triple layer $\mathrm{CrI}_{3} / \mathrm{WSe}_{2}$ heterostructures, the interlayer interaction tuned the band gap to indirect (without considering SOC). When considering the SOC, tri-Se still have indirect band gap, in which the valence band maximum (VBM) is located at $\Gamma$ point, but the conduction band minimum (CBM) is located at $\mathrm{K}$ point (see Fig. 5). As shown in Fig. 4 and 5, less than $0.1 \mathrm{eV}$ energy difference at different $\mathrm{K}$ points in the flat conductance bands. Then, it is easier to switch between the direct band gap and indirect one by using different interlayer interactions. It is found that the CBM is contributed by Cr, I and $\mathrm{W}$ atoms, then the SOC effect mainly resulting from $\mathrm{I}^{-}$ atoms is one reason for the direct to indirect change.

Magnetic anisotropy originating mainly from SOC effects, ${ }^{17,30}$ is an important parameter when it comes to $2 \mathrm{D}$ magnets as it is qualitatively related to their magnetic stability. MAE is defined as the difference between energies corresponding to the magnetization in the in-plane and out-plane directions ( $\mathrm{MAE}=$ $E_{\mathrm{m} / / \mathrm{a}}-E_{\mathrm{m} / \mathrm{c} \mathrm{c}}$ ), in which $E_{\mathrm{m} / / \mathrm{a}}$ is the energy for the in-plane magnetization, and $E_{\mathrm{m} / / \mathrm{c}}$ is the energy for the out-plane magnetization. A positive (negative) value of MAE indicated the out-plane (in-plane) easy axis. Taking SOC effects into account, the total energies $E_{\mathrm{m} / / \mathrm{a}}$ and $E_{\mathrm{m} / \mathrm{c}}$ can be achieved through noncollinear calculations, and then the MAE can be evaluated. Consistent with the previous calculations ${ }^{12,18,19,21}$ and experimental results, ${ }^{13,17}$ because of the strong SOC in the heavier iodine ions,${ }^{17}$ the easy axis for monolayer $\mathrm{CrI}_{3}$ is outplane with MAE $0.73 \mathrm{meV}$ per $\mathrm{Cr}$ atom.

One most interesting finding is that, as shown in Table 2, the out-plane easy axis in monolayer $\mathrm{CrI}_{3}$ changed into in-plane in $\mathrm{CrI}_{3} / \mathrm{WSe}_{2}$ heterostructures with almost all kinds of stacking types. The only exception is tri-T, which is still out-plane easy 

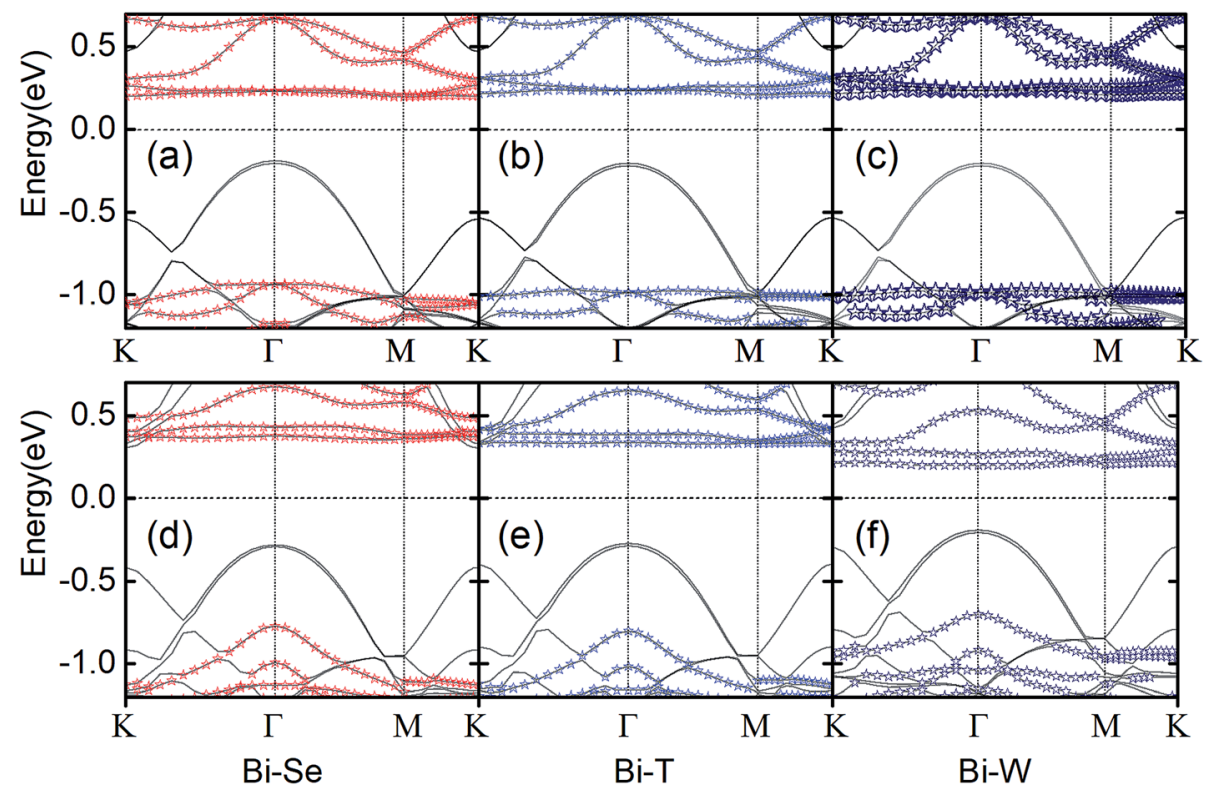

Fig. 4 Band structure of bilayer $\mathrm{Crl}_{3} / \mathrm{WSe}_{2}$ heterostructures without ( $(\mathrm{a}-\mathrm{c}$ ) and with (d-f) SOC for bi-Se (a and d), bi-T (b and e) and bi-W (c and f) stackings. The grey lines are band of the heterostructure, and stars are band mainly contributed from $\mathrm{Crl}_{3}$ layer. The bandgap is indirect without SOC, but tuned direct for bi-T and bi-W stackings after the SOC have been considered.

axis with MAE $0.03 \mathrm{meV}$ per Cr. Remarkable large MAE can be found for the most stable stacking configurations in both bilayer and triple layer $\mathrm{CrI}_{3} / \mathrm{WSe}_{2}$ heterostructures, i.e., 0.17 $\mathrm{meV}$ per $\mathrm{Cr}$ and $0.23 \mathrm{meV}$ per $\mathrm{Cr}$ for bi-Se and tri-Se, respectively.

Noteworthy, the charge transfer in bilayer heterostructures (see Table 1) can result in a net dipole moment. With the dipole correction, our calculation indicated that the MAE and magnetic moment can be changed about $0.001 \mathrm{meV}$ per $\mathrm{Cr}$ and
$0.001 \mu_{\mathrm{B}}$ per Cr, respectively. In Fig. $6 \mathrm{a}$, take bi-Se $\mathrm{CrI}_{3} / \mathrm{WSe}_{2}$ heterostructures as an example, we compared the wave function characteristics at the $\mathrm{G}$ point $\mathrm{Cr} \mathrm{d}$ and $\mathrm{Se} \mathrm{p}_{z}$ orbits between outplane and in-plane easy axis. It can be seen clearly that, the hybridizations take placed between $\mathrm{Cr}_{-} \mathrm{d}_{x z, y z}, \mathrm{Cr}_{-} \mathrm{d}_{z}{ }^{2}$ and $\mathrm{Se} \_\mathrm{p}_{z}$ orbits, as the results of the interlayer interaction between $\mathrm{CrI}_{3}$ layer and $\mathrm{WSe}_{2}$ layer. This should responsible for the easy axis transition in $\mathrm{CrI}_{3} / \mathrm{WSe}_{2}$ heterostructures as suggested in $\mathrm{Fe} /$ MgO interfaces. ${ }^{52}$ The only exception is the tri- $\mathrm{T} \mathrm{CrI}_{3} / \mathrm{WSe}_{2}$
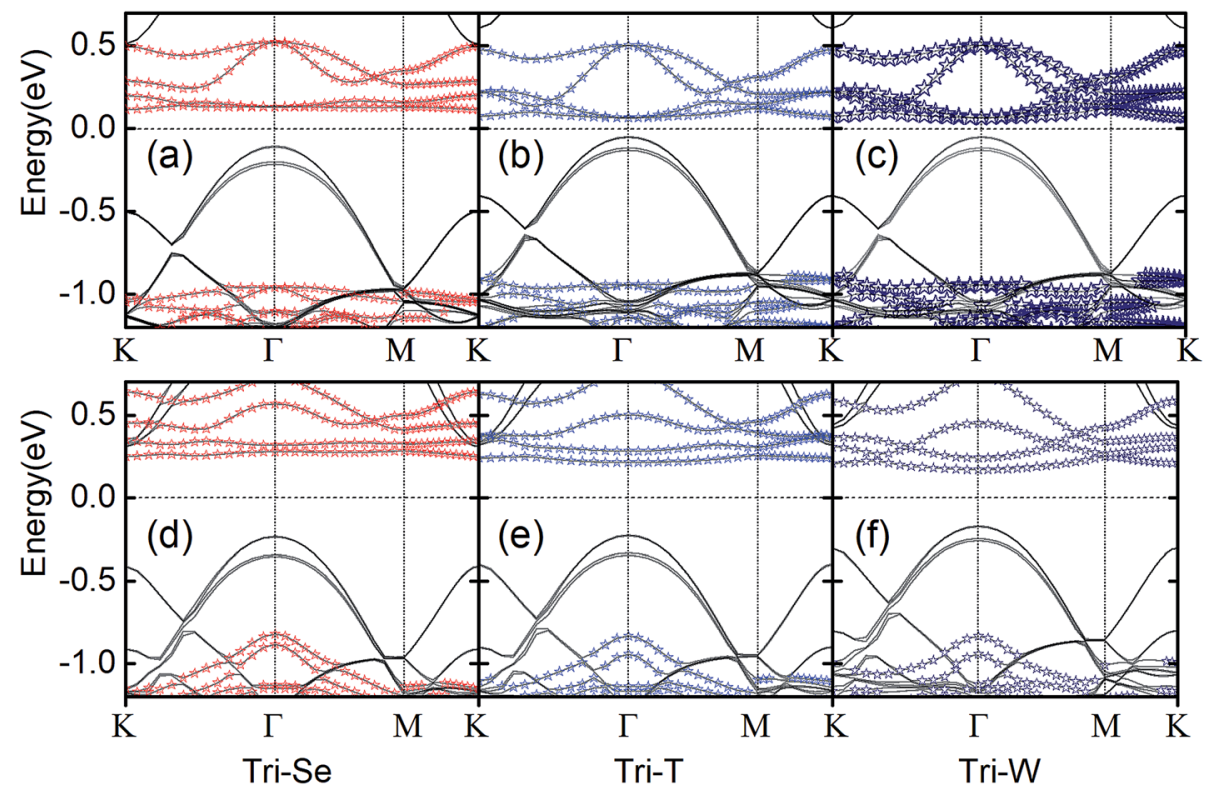

Fig. 5 Band structure of triple layer $\mathrm{Crl}_{3} / \mathrm{WSe}_{2}$ heterostructures without (a-c) and with (d-f) SOC for tri-Se (a and d), tri-T (b and e) and tri-W (c and f) stackings. The grey lines are band of the heterostructure, and stars are band mainly contributed from $\mathrm{Crl}_{3} \mathrm{layer}_{\mathrm{f}}$ 
Table 2 The magnetization directions for bi-layer and tri-layer $\mathrm{Crl}_{3} /$ $\mathrm{WSe}_{2}$ heterostructures. The MAE (in units of meV per $\mathrm{Cr}$ ) is also listed in the bracket, a positive (negative) value indicated the out-plane (inplane) easy axis

\begin{tabular}{llll}
\hline & -Se & $-\mathrm{T}$ & $-\mathrm{W}$ \\
\hline Monolayer & Out-plane $(0.73)$ & & \\
$\mathrm{Bi}$ & In-plane $(-0.17)$ & In-plane $(-0.03)$ & In-plane $(-0.02)$ \\
Tri & In-plane $(-0.23)$ & Out-plane $(0.03)$ & In-plane $(-0.04)$
\end{tabular}

heterostructure, in which the out-plane easy axis is maintained. Therefore, except for applying the in-plane external magnetic field,$^{18}$ we demonstrated here that the spin direction can be shifted from out-plane to in-plane by stacking with the $\mathrm{WSe}_{2}$ layer. The easy axis direction should be another reason for the direct-to-indirect band gap transition. We calculated the band structures for bi-Se $\mathrm{CrI}_{3} / \mathrm{WSe}_{2}$ heterostructure with two different magnetic moment orientations $(\mathrm{m} / / \mathrm{a}$ and $\mathrm{m} / / \mathrm{c}$ ) as shown in Fig. $6 \mathrm{~b}$. For $\mathrm{m} / \mathrm{a}$, the CBM located at $\mathrm{K}$ point, but the VBM is located at $\Gamma$ point, which makes the band gap indirect. For $\mathrm{m} / / \mathrm{c}$, however, both the CBM and VBM located at $\Gamma$ point, then the band gap is direct. Moreover, it can be also found in Fig. $6 \mathrm{~b}$ and $4 \mathrm{a}$, both the CBM and VBM are degenerated at $\Gamma$ point for $\mathrm{m} / /$
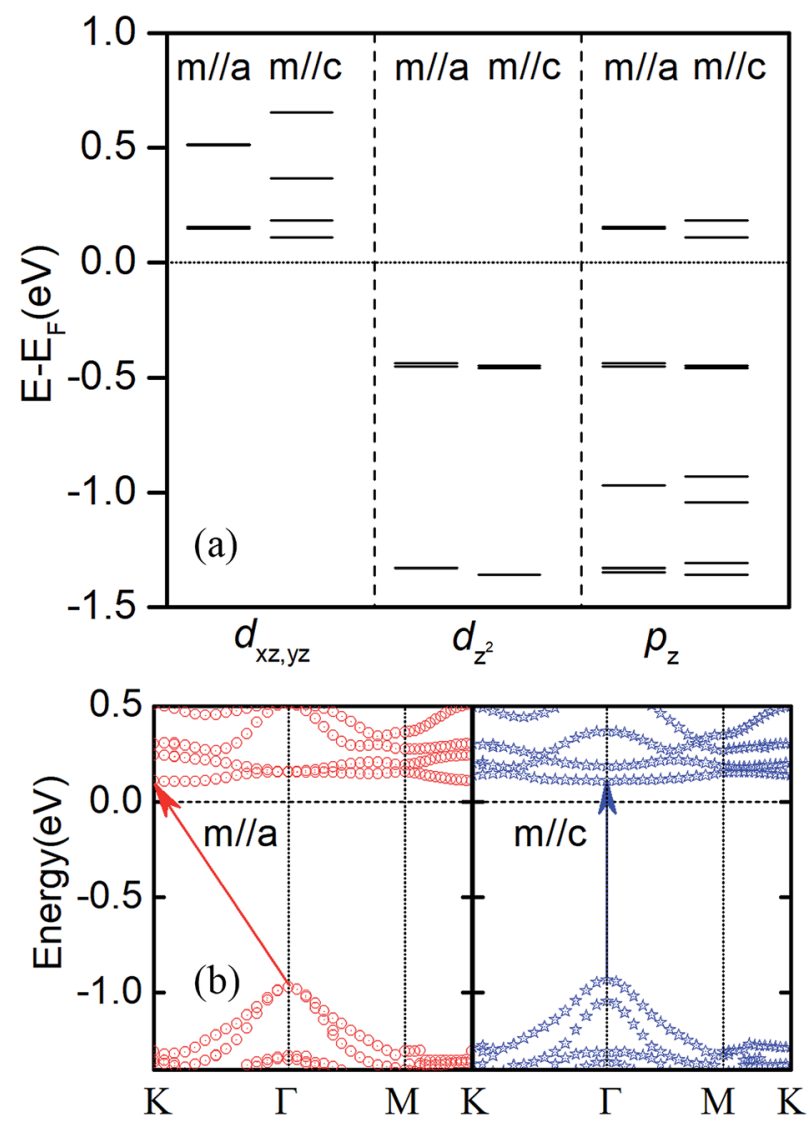

Fig. 6 (a) Effects of spin axis on the wave function characteristics at $G$ points for $\mathrm{Cr} d$ and Se $\mathrm{p}_{z}$ orbitals for bi-Se $\mathrm{Crl}_{3} / \mathrm{WSe}_{2}$ heterostructures. (b) Band structure of bi-Se $\mathrm{Crl}_{3} / \mathrm{WSe}_{2}$ heterostructures for the magnetic moment along the in-plane $a$ axis (left panel) out-of-plane $c$ axis (right panel). The arrows show that the bandgap is indirect with $\mathrm{m} / / \mathrm{a}$ and direct with $\mathrm{m} / / \mathrm{c}$. a and without SOC. It is known that, the CBM at $\Gamma$ point is mainly results from $\mathrm{p}_{x}$ and $\mathrm{p}_{y}$ orbitals of I atom. ${ }^{18}$ When the SOC is included and with the out-plane easy axis, the energy split for VBM and CBM at $\Gamma$ point. Therefore, the transition of easy axis from out-plane to in-plane is one reason for the direct-toindirect band gap switch.

\section{Conclusions}

To summarize, our first-principles calculations unveil the effect of interfaces on the electronic and magnetic properties of vertical $\mathrm{CrI}_{3} / \mathrm{WSe}_{2}$ heterostructures. We find that the -Se stacking is the most stable configuration in either bilayer or triple layer $\mathrm{CrI}_{3} / \mathrm{WSe}_{2}$ heterostructures. The switch between direct and indirect band gap can be found mainly because of the SOC effects of the p orbits of I atoms. Most importantly, originated from hybridizations between $\mathrm{d}$ orbits of $\mathrm{Cr}$ atoms and $\mathrm{p}$ orbital of Se atoms, the easy axis transition from the out-plane of monolayer $\mathrm{CrI}_{3}$ to in-plane for almost all stacking type of both bilayer and triple layer $\mathrm{CrI}_{3} / \mathrm{WSe}_{2}$ heterostructures is found. All these theoretical results constitute a useful picture of vertical $\mathrm{CrI}_{3} / \mathrm{WSe}_{2}$ heterostructures for the interface effect on the electronic and magnetic behaviors and provide vital guidance in designing novel spintronic $2 \mathrm{D}$ devices.

\section{Conflicts of interest}

There are no conflicts to declare.

\section{Acknowledgements}

This work was supported by the National Natural Science Foundation of China (11674046) and the Scientific and Technological Innovation Programs of Higher Education Institutions in Shanxi (201802070).

\section{Notes and references}

1 A. K. Geim and K. S. Novoselov, Nat. Mater., 2007, 6, 183.

2 K. S. Novoselov, A. K. Geim, S. V. Morozov, D. Jiang, Y. Zhang, S. V. Dubonos, I. V. Grigorieva and A. A. Firsov, Science, 2004, 306, 666-669.

3 Y. Kubota, K. Watanabe, O. Tsuda and T. Taniguchi, Science, 2007, 317, 932-934.

4 H. Fang, S. Chuang, T. C. Chang, K. Takei, T. Takahashi and A. Javey, Nano Lett., 2012, 12, 3788-3792.

5 K. F. Mak, C. Lee, J. Hone, J. Shan and T. F. Heinz, Phys. Rev. Lett., 2010, 105, 136805.

6 G. A. Prinz, Science, 1998, 282, 1660.

7 W. Han, R. K. Kawakami, M. Gmitra and J. Fabian, Nat. Nanotechnol., 2014, 9, 794.

8 C. Gong, L. Li, Z. Li, H. Ji, A. Stern, Y. Xia, T. Cao, W. Bao, C. Wang, Y. Wang, Z. Q. Qiu, R. J. Cava, S. G. Louie, J. Xia and X. Zhang, Nature, 2017, 546, 265.

9 B. Huang, G. Clark, E. Navarro-Moratalla, D. R. Klein, R. Cheng, K. L. Seyler, D. Zhong, E. Schmidgall, 
M. A. McGuire, D. H. Cobden, W. Yao, D. Xiao, P. JarilloHerrero and X. Xu, Nature, 2017, 546, 270.

10 M. Bonilla, S. Kolekar, Y. Ma, H. C. Diaz, V. Kalappattil, R. Das, T. Eggers, H. R. Gutierrez, M.-H. Phan and M. Batzill, Nat. Nanotechnol., 2018, 13, 289-293.

11 J. F. Dillon Jr and C. E. Olson, J. Appl. Phys., 1965, 36, 12591260.

12 W.-B. Zhang, Q. Qu, P. Zhu and C.-H. Lam, J. Mater. Chem. C, 2015, 3, 12457-12468.

13 M. A. McGuire, H. Dixit, V. R. Cooper and B. C. Sales, Chem. Mater., 2015, 27, 612-620.

14 L. Chen, J.-H. Chung, B. Gao, T. Chen, M. B. Stone, A. I. Kolesnikov, Q. Huang and P. Dai, Phys. Rev. X, 2018, 8, 041028.

15 C. Lee, F. Katmis, P. Jarillo-Herrero, J. S. Moodera and N. Gedik, Nat. Commun., 2016, 7, 12014.

16 J. Zhang, B. Zhao, T. Zhou, Y. Xue, C. Ma and Z. Yang, Phys. Rev. B, 2018, 97, 085401.

17 J. L. Lado and J. Fernández-Rossier, 2D Materials, 2017, 4, 035002.

18 P. Jiang, L. Li, Z. Liao, Y. X. Zhao and Z. Zhong, Nano Lett., 2018, 18, 3844-3849.

19 L. Webster and J.-A. Yan, Phys. Rev. B, 2018, 98, 144411.

20 F. Iyikanat, M. Yagmurcukardes, R. T. Senger and H. Sahin, J. Mater. Chem. C, 2018, 6, 2019-2025.

21 G. Guo, G. Bi, C. Cai and H. Wu, J. Phys.: Condens. Matter, 2018, 30, 285303.

22 F. Zheng, J. Zhao, Z. Liu, M. Li, M. Zhou, S. Zhang and P. Zhang, Nanoscale, 2018, 10, 14298-14303.

23 Z. Wu, J. Yu and S. Yuan, Phys. Chem. Chem. Phys., 2019, 21, 7750-7755.

24 J. Liu, M. Shi, J. Lu and M. P. Anantram, Phys. Rev. B, 2018, 97, 054416.

25 S. Jiang, L. Li, Z. Wang, K. F. Mak and J. Shan, Nat. Nanotechnol., 2018, 13, 549-553.

26 B. Huang, G. Clark, D. R. Klein, D. MacNeill, E. NavarroMoratalla, K. L. Seyler, N. Wilson, M. A. McGuire, D. H. Cobden, D. Xiao, W. Yao, P. Jarillo-Herrero and X. Xu, Nat. Nanotechnol., 2018, 13, 544-548.

27 N. Sivadas, S. Okamoto, X. Xu, C. J. Fennie and D. Xiao, Nano Lett., 2018, 18, 7658-7664.

28 M. U. Farooq and J. Hong, NPJ. 2D Mater. Appl., 2019, 3, 3.

29 Z. Wang, I. Gutiérrez-Lezama, N. Ubrig, M. Kroner, M. Gibertini, T. Taniguchi, K. Watanabe, A. Imamoğlu, E. Giannini and A. F. Morpurgo, Nat. Commun., 2018, 9, 2516.

30 A. K. Geim and I. V. Grigorieva, Nature, 2013, 499, 419.

31 Y. Liu, N. O. Weiss, X. Duan, H.-C. Cheng, Y. Huang and X. Duan, Nat. Rev. Mater., 2016, 1, 16042.

32 M. P. Levendorf, C.-J. Kim, L. Brown, P. Y. Huang, R. W. Havener, D. A. Muller and J. Park, Nature, 2012, 488, 627.
33 Y. Gong, J. Lin, X. Wang, G. Shi, S. Lei, Z. Lin, X. Zou, G. Ye, R. Vajtai, B. I. Yakobson, H. Terrones, M. Terrones, B. K. Tay, J. Lou, S. T. Pantelides, Z. Liu, W. Zhou and P. M. Ajayan, Nat. Mater., 2014, 13, 1135.

34 M.-Y. Li, Y. Shi, C.-C. Cheng, L.-S. Lu, Y.-C. Lin, H.-L. Tang, M.-L. Tsai, C.-W. Chu, K.-H. Wei, J.-H. He, W.-H. Chang, K. Suenaga and L.-J. Li, Science, 2015, 349, 524-528.

35 J. Zhang, W. Xie, J. Zhao and S. Zhang, 2D Materials, 2016, 4, 015038.

36 J. Zhang, W. Xie, M. L. Agiorgousis, D.-H. Choe, V. Meunier, X. Xu, J. Zhao and S. Zhang, Nanoscale, 2018, 10, 7912-7917.

37 K. D. Pham, N. N. Hieu, H. V. Phuc, B. D. Hoi, V. V. Ilysov, B. Amin and C. V. Nguyen, Comput. Mater. Sci., 2018, 153, 438-444.

38 P. T. T. Le, L. M. Bui, N. N. Hieu, H. V. Phuc, B. Amin, N. V. Hieu and C. V. Nguyen, Diamond Relat. Mater., 2019, 94, 129-136.

39 K. D. Pham, N. N. Hieu, H. V. Phuc, I. A. Fedorov, C. A. Duque, B. Amin and C. V. Nguyen, Appl. Phys. Lett., 2018, 113, 171605.

40 A. I. Buzdin, Rev. Mod. Phys., 2005, 77, 935-976.

41 A. H. MacDonald, P. Schiffer and N. Samarth, Nat. Mater., 2005, 4, 195.

42 D. Zhong, K. L. Seyler, X. Linpeng, R. Cheng, N. Sivadas, B. Huang, E. Schmidgall, T. Taniguchi, K. Watanabe, M. A. McGuire, W. Yao, D. Xiao, K.-M. C. Fu and X. Xu, Sci. Adv., 2017, 3, e1603113.

43 K. L. Seyler, D. Zhong, B. Huang, X. Linpeng, N. P. Wilson, T. Taniguchi, K. Watanabe, W. Yao, D. Xiao, M. A. McGuire, K.-M. C. Fu and X. Xu, Nano Lett., 2018, 18, 3823-3828.

44 G. Kresse and D. Joubert, Phys Rev B, 1999, 59, 1758-1775.

45 G. Kresse and J. Furthmüller, Comput. Mater. Sci., 1996, 6, 15-50.

46 J. P. Perdew, K. Burke and M. Ernzerhof, Phys. Rev. Lett., 1996, 77, 3865-3868.

47 S. Grimme, J. Antony, S. Ehrlich and H. Krieg, J. Chem. Phys., 2010, 132, 154104.

48 H. J. Monkhorst and J. D. Pack, Phys. Rev. B: Solid State, 1976, 13, 5188-5192.

49 S. L. Dudarev, G. A. Botton, S. Y. Savrasov, C. J. Humphreys and A. P. Sutton, Phys. Rev. B: Condens. Matter Mater. Phys., 1998, 57, 1505-1509.

50 M. Dienwiebel, G. S. Verhoeven, N. Pradeep, J. W. M. Frenken, J. A. Heimberg and H. W. Zandbergen, Phys. Rev. Lett., 2004, 92, 126101.

51 G. Henkelman, A. Arnaldsson and H. Jónsson, Comput. Mater. Sci., 2006, 36, 354-360.

52 H. X. Yang, M. Chshiev, B. Dieny, J. H. Lee, A. Manchon and K. H. Shin, Phys. Rev. B: Condens. Matter Mater. Phys., 2011, 84, 054401. 